\title{
Digitalisierung der Arbeitssicherheit auf Baustellen
}

Jochen Teizer, Markus König, Thomas Herrmann, Markus Jelonek, Stephan Embers, Caner Kazanci, Samed Bükrü, Olga Golovina, Ian Quirke, Edgar Glasner, Oliver Daum, Jens Richter, Jochen Hanff, Eleni Kikidi, Daniel Hecker, Raimo Vollstädt und Wolfgang Bücken

\subsection{Vorstellung der mitwirkenden Projektpartner}

DigiRAB ist ein Forschungs- und Entwicklungsprojekt der Verbundpartner RuhrUniversität Bochum, Ed. Züblin AG, Selectronic Funk- und Sicherheitstechnik $\mathrm{GmbH}$, thinkproject Deutschland $\mathrm{GmbH}$, Topcon Deutschland Positioning GmbH. Als Assoziierte Partner nehmen die Berufsgenossenschaft Bau und die Hilti AG teil. DigiRAB wurde im Rahmen des Programms ,Innovationen für die Produktion, Dienstleistung und Arbeit"/,Zukunft der Arbeit" vom Bundesministerium für Bildung und Forschung (BMBF) und dem Europäischen Sozialfonds (ESF) gefördert und vom Projektträger Karlsruhe (PTKA) betreut.

J. Teizer $(\bowtie) \cdot$ M. König $\cdot$ S. Embers $\cdot$ C. Kazanci $\cdot$ S. Bükrü $\cdot$ O. Golovina Ruhr-Universität Bochum, Informatik im Bauwesen, Bochum, Deutschland

T. Herrmann $\cdot$ M. Jelonek

Ruhr-Universität Bochum, Informations- und Technikmanagement, Bochum, Deutschland

I. Quirke · E. Glasner · O. Daum

Ed. Züblin AG - Dirketion Mitte, Frankfurt, Deutschland

J. Richter

Selectronic Funk- und Sicherheitstechnik GmbH, Hünstetten, Deutschland

J. Hanff · E. Kikidi · D. Hecker

thinkproject GmbH, Essen, Deutschland

R. Vollstädt · W. Bücken

Topcon Deutschland Positioning GmbH, Hamburg, Deutschland

(C) Der/die Autor(en) 2021

W. Bauer et al. (Hrsg.), Arbeit in der digitalisierten Welt,

https://doi.org/10.1007/978-3-662-62215-5_26 


\subsection{Zielsetzung}

Arbeitnehmer und Arbeitnehmerinnen auf Baustellen sind höheren Gefahren ausgesetzt als in vielen anderen Industrien. $\mathrm{Zu}$ den schwerwiegendsten Unfallursachen gehören das Abstürzen aus Höhen, das Arbeiten im Umfeld von Baumaschinen und der unsachgemäße Umgang mit handgeführten Geräten. Für die Bauunternehmen führen Arbeitsunfälle und Berufskrankheiten infolge von Bauverzögerungen und Schadensersatzansprüchen zu deutlichen Mehrkosten. Erschwerend hinzu kommen eine zunehmende Komplexität der Bauprojekte und neue technische und ökonomische Herausforderungen. In immer kürzerer Zeit soll eine hohe Bauqualität gewährleistet werden [24].

Die Digitalisierung im Bauwesen hat in den letzten Jahren zu neuen Arbeitsweisen geführt. Für die Planung und Umsetzung des Arbeitsschutzes in Bauprojekten werden die digitalen Möglichkeiten aktuell noch nicht konsequent genutzt. Im Verbundprojekt „DigiRAB - Sicheres Arbeiten auf der digitalisierten Baustelle” wurden daher sowohl smarte Prozesse als auch Technologien für das sichere Arbeiten auf Baustellen entwickelt und getestet.

Der „Projektdemonstrator DigiRAB“ ist ein virtuelles Bauprojekt, das als Vehikel genutzt wird, um nachvollziehbar und baustellennah die Ergebnisse des Projektes DigiRAB zu beschreiben. Der entwickelte Projektdemonstrator stellt ein zusammenhängendes Szenario dar, das die Handlungsbereiche (HB) „Sicher Planen“ (HB 1), „Proaktiv Warnen“ (HB 2) und „Personalisiert Lernen und Schulen“ (HB 3) erläutert und miteinander verbindet (Abb. 26.1). Als verbindendes Element zwischen den Handlungsbereichen 1 und 2 dient die BIM Management Software DESITE MD vom Projektpartner thinkproject Deutschland GmbH. BIM Modelle aus HB 1 und 2 können

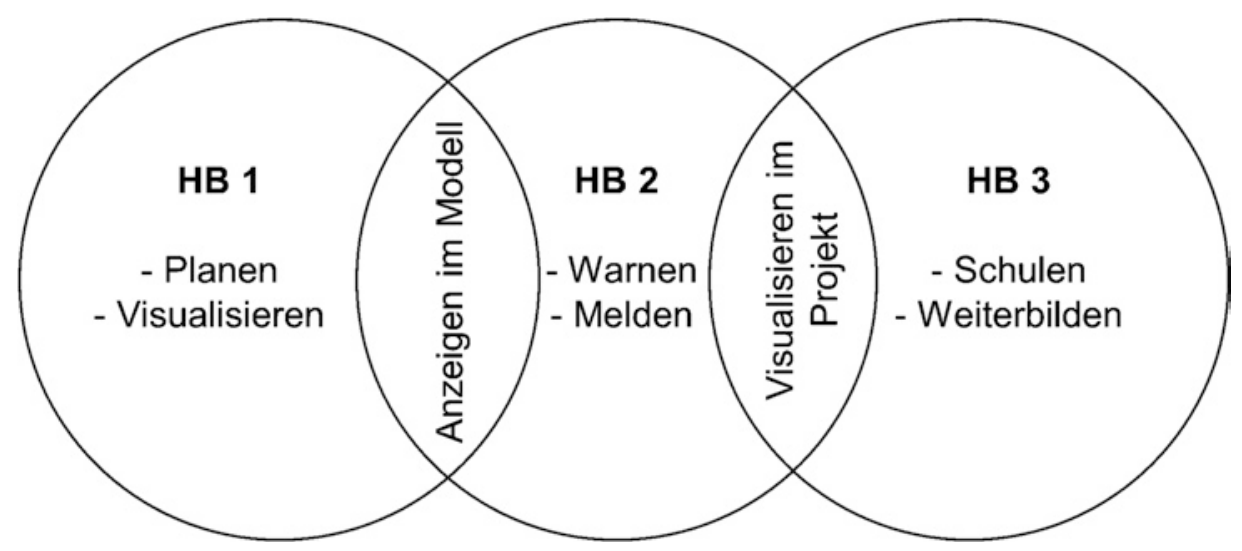

Abb. 26.1 Darstellung der Handlungsbereiche und deren Zusammenhänge. (Quelle: Eigene Abbildung) 
zu Schulungszwecke in HB 3 - mit und ohne konkreten Projektbezug - verwendet werden.

\subsection{Digitale Planung und Präventive Unfallvermeidung (HB1)}

\subsubsection{Ausgangslage}

Vor Baubeginn gilt es, mit den ausführenden Unternehmen den anstehenden Bauprozess zu analysieren und dessen Gefährdungen zu beurteilen. Hierfür werden in der Gefährdungsbeurteilung auch einzusetzende Schutzmaßnahmen geplant. Unter projektspezifischen Randbedingungen ist die räumliche und zeitliche Organisation des Arbeitsschutzes gewerkeübergreifend in einem Sicherheits- und Gesundheitsschutzplan (SiGe Plan) festzuhalten. Der Planungsprozess der Arbeitssicherheit erfolgt derzeit weitestgehend ohne digitale Prozessunterstützung und ist oft von baubetrieblichen Belangen entkoppelt. Bei dieser Vorgehensweise entstehen deshalb z. T. unabgestimmte Handlungsanweisungen. Oftmals sind der genaue Zeitpunkt, die Menge und die Art der notwendigen Schutzeinrichtung oder -maßnahme nicht klar definiert.

\subsubsection{Motivation und Zielsetzung}

Das Hauptziel im HB1 des Forschungsprojektes DigiRAB war die Untersuchung inwieweit digitale Werkzeuge und alternative Arbeitsmethoden den Arbeitsschutzplanenden eine Unterstützung dabei bieten, den Arbeitsschutz bei komplexen schlüsselfertigen Bauprojekten detaillierter und adaptiver gestalten zu können. Dabei sollen die bestehenden Prozesse der Gefährdungsbeurteilung so unterstützt werden, dass umfangreiche arbeitsschutztechnische Handlungsanweisungen mit niedrigem Zeitaufwand an das Baustellenpersonal gestellt werden können. Darüber hinaus sollten die „Bausteine“ (technische Lösungen und Sicherheitshinweise zur Vermeidung von Unfällen und Gesundheitsgefahren) der Berufsgenossenschaft Bau (BG Bau) Verwendung finden.

\subsubsection{Digitale Planung}

Das Bauwesen in Deutschland befindet sich seit einigen Jahren im Umbruch, vor allen Dingen bei Veränderungen in den baustellennahen Prozessen. Die zunehmende Verbreitung von Lean Construction und Building Information Modeling (BIM) bietet eine Chance bestehende Prozesse besser aufeinander abzustimmen sowie den dynamischen Bauprozess vorausschauender und flexibler zu gestalten [1]. Mit Hilfe von Lean Construction werden alle Bauprozesse vorausschauend und möglichst mit allen Prozessbeteiligten vor allem hinsichtlich Ressourcenschonung (Mensch, Material und Zeit) ana- 
lysiert. Hierbei steht zu Beginn (in der Regel bauvorbereitend) die Prozessplanung im Vordergrund, während des Bauablaufs spielt die Prozesssteuerung die zentrale Rolle.

Die konsequente Anwendung der BIM Methodik erlaubt die Transformation der „klassischen“ Architektenzeichnungen in dreidimensionale digitale Bauwerksmodelle, in dem sämtliche relevanten Informationen aller Projektbeteiligten zur gemeinschaftlichen Nutzung zentral zusammengeführt werden - von den zeitlichen Abläufen (4D) bis hin zu benötigten Materialien, Bestellmengen und Betreiberdaten (5D). Der Projektpartner Ed. Züblin AG strebt mit BIM 5D ${ }^{\circledR}$ eine Umsetzung dieser Entwicklung an. Die Koppelung der Vorgehensweise von Lean Construction mit BIM findet zunehmend statt. HB 1 ist auf diesen Grundlagen konzipiert. Das bedeutet, dass alle Planungsschritte in der Angebots- und Bauvorbereitungsphase eines Bauprojektes auf der Grundlage von BIM erfolgen und allen Baubeteiligten zur Verfügung gestellt werden können.

\subsubsection{Vorgehensweise}

Der Fokus wurde auf die Phase der Bauvorbereitung gelegt, in der bereits konkrete Schritte für die Abwicklung des Bauprojektes unternommen werden. Ein BIM Modell - also das digitale Abbild des Bauvorhabens - besitzt in dieser Projektphase vor allem planerische Inhalte zum Bausoll und wenige Inhalte zur Bauvorbereitung und -abwicklung. Das im DigiRAB-Projekt verwendete BIM Modell wurde mit den mit Lean Construction Methoden entwickelten Terminplan zu einem 4D Modell verknüpft und bildet den Ausgangspunkt für die digitale Planung des Arbeitsschutzes. In diesem Zusammenhang wurden in HB 1 Werkzeuge entwickelt, um teilautomatisch BIM Inhalte für eine sichere Bauabwicklung zu erzeugen und als Entscheidungsgrundlage für die Baubeteiligten zu liefern. Mittels einer detaillierten Analyse des allgemeinen Bauprozesses wurden die relevanten Aktivitäten zur Gewährleistung von Sicherheit identifiziert, wie zum Beispiel Optimierung der Logistik, Aktualisierung von SiGe-Plänen, Unterweisung und Schulung, Begehung und Identifizierung kritischer Stellen. Für identifizierte Aktivitäten ergibt sich die Anforderung, sie weitgehend durch BIM zu unterstützen, indem etwa Gefahrenpunkte sensorgestützt erkannt und im Modell markiert werden.

\subsubsection{Ergebnisse}

Das erste Ergebnis ist ein digitales Werkzeug, ein sogenannter „Rule-Checking“Algorithmus [2-4] (weiterentwickelt durch den Projektpartner Ruhr-Universität Bochum), das bestehende BIM Modelle analysiert und dem Anwender regelbasiert Vorschläge für verschiedene Arbeitssicherheitsmaßnahmen unterbreitet. Diese Vorschläge können z. B. 


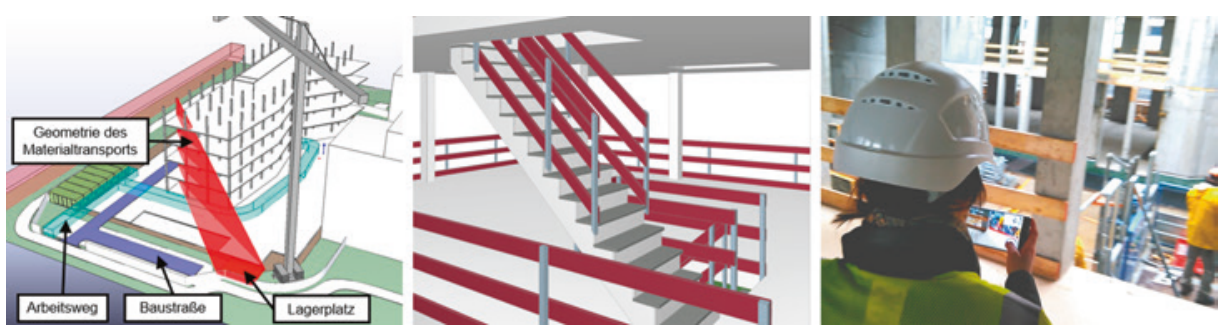

Abb. 26.2 Digitale Arbeitsvorbereitung und Anzeigen auf Baustellen [4]

Absturzsicherungen sein (Abb. 26.2). Ist ein Terminplan mit dem Modell verknüpft (4D), so haben die Vorschläge eine Zeitkomponente für verschiedene Bauphasen. Auf dieser Grundlage können verlässliche Pläne für Ein- und Umbau der Absturzsicherung erstellt werden und so auch eine digitale Kontrolle des Soll-Zustands erfolgen.

In einer Erweiterung des Rule-Checking wurde auch untersucht, ob Schwenkbereiche von Lasten unter Baukräne sowie Lastausbreitung unter Maschinenaufstandsflächen abgebildet werden können, um Gefahrenbereiche schneller zu erkennen und diese bei der Planung der Baustelleneinrichtung zu berücksichtigen. Das Rule-Checking wurde in der BIM Management Software DESITE MD implementiert. In DESITE MD wurde auch das zweite Ergebnis von HB 1 entwickelt, die teilautomatische Erstellung eines sogenannten „4D-SiGe-Plans“ inklusive digitaler Gefährdungsbeurteilung. Hierzu können in der Software zunächst Dokumente zu Gefährdungspotenzialen unter Einbindung der Bausteine des BG BAU hinterlegt werden. Diese können dann mit sog. Prozessbausteinen, die als Vorlage in der Software hinterlegt werden, verknüpft werden. Die Prozessbausteine legen fest, welche Aktivitäten für die Erstellung eines Bauteils durchzuführen sind und definieren somit die einzelnen Arbeitsinhalte. Auf Basis der Prozessbausteine kann in Verbindung mit der Gebäudestruktur ein erster Entwurf der Terminplanung automatisch berechnet werden. Hierbei werden die Dokumente zu den Gefährdungspotenzialen automatisiert mit den Vorgängen des Terminplans verknüpft. Für jeden Vorgang können anschließend die relevanten Gefährdungspotenziale angezeigt werden.

Alternativ können die Dokumente zu Gefährdungspotenzialen mit den Vorgängen eines vorhandenen 4D-Terminplans händisch verknüpft werden. In beiden Fällen liegt als Arbeitsergebnis eine Gefährdungsbeurteilung in einem zeitlichen Rahmen, also ein 4D-SiGe-Plan vor $[22,23]$. Als weiteres Ergebnis von HB 1 wurde eine sichere Planung der Baustelleneinrichtung nach Lean Prinzipien weiterentwickelt: Unter Einbeziehung des Rule-Checking und des 4D-SiGe-Plans können die Baustelleneinrichtungspläne auch in ihrer notwendigen zeitlichen Fortentwicklung einfacher erstellt und den Anwendern mithilfe des BIM Modells schneller vermittelt werden. 


\subsection{Proaktiv Warnen, Melden und Auswerten von Daten (HB 2)}

\subsubsection{Ausgangslage}

Die Zahl der jährlich tödlich verunglückten Personen auf Baustellen weltweit wird auf mindestens 60.000 geschätzt [5]. Auch in Deutschland ist die Anzahl der meldepflichtigen Arbeitsunfälle im Baubereich in den letzten Jahren leicht gestiegen. Damit sind Todesfälle und Verletzungen in der Bauindustrie im Vergleich zu anderen Branchen immer noch überdurchschnittlich hoch. Rund ein Viertel aller tödlichen Unfälle haben direkten Bezug zu Arbeiten mit oder in der Nähe von Baumaschinen [6]. Dabei können die Ursachen vielfältig sein: Eingeschränktes Sichtfeld, Rückwärtsfahren, schlechte Umgebungsbedingungen (Wetter, Sicht, usw.) und ein sich stetig veränderndes Baufeld [7].

\subsubsection{Motivation und Zielsetzung}

Aktuell bestehende Systeme zur Umfelderkennung bei Baumaschinen beschränken sich auf die Erkennung und Warnung im Gefahrenfall. Eine Aufzeichnung - geschweige denn Auswertung der Häufigkeit - sowohl zeitlich als auch lokal - besteht bisher nicht [8]. Daher können auch keinerlei Rückschlüsse auf vorhandene Gefahrenpotenziale geschlossen werden, die in vorausschauenden und vorbeugenden Maßnahmen Anwendung finden könnten.

Der Ansatz des Projektes DigiRAB ist, Gefahrenpotenziale durch das Erkennen und Melden potenzieller Gefährdungssituationen frühzeitig zu erkennen, um rechtzeitig Präventionsmaßnahmen einleiten zu können. Daher strebt DigiRAB eine Echtzeitübermittlung und -visualisierung der erfolgten Beinaheunfälle, sowohl zwischen Baumaschinen untereinander als auch zwischen Baumaschinen und Personal am Boden an. Dabei soll ein Erkennungssystem zum Einsatz kommen, welches nicht auf optischer Sensorik beruht. Hiermit sollen Unwägbarkeiten wie tote Winkel, nicht einsehbare Bereiche wie beispielsweise Gebäudeecken, Mauern und Baumaterial ausgeschlossen werden. Die erhobenen Informationen sollen in Echtzeit über einen Webservice auf einen Datenserver übertragen werden. Mittels der BIM Management Software DESITE MD können die Daten vom Server abgerufen und im BIM Modell dokumentiert, visualisiert und analysiert werden, um weisungsbefugten Entscheidungsträgern auf der Baustelle (Bauleiter, Fachkraft für Arbeitssicherheit und weitere) verlässliche Informationen an die Hand zu geben, wo Gefahrenpotenziale in der Praxis bestehen, wo diese sich häufen, und wie diese ggfs. abzustellen sind [9]. 


\subsubsection{Stand der Technik}

Derzeitige Systeme zur Umgebungserkennung von Baumaschinen basieren heute vornehmlich auf optischen Sensoren: Kameras, Stereokameras, Laserscannern oder eine Kombination aus diesen [9, 10]. Eine zweifelsfreie und unter den verschiedenen Umgebungs- und Wetterbedingungen zuverlässige Erkennung von Personen in der Umgebung von Baumaschinen, Baumaschinen untereinander oder sonstigen baustellenbedingten Hindernissen im Umgebungsbereich der Baumaschine ist mit diesen Technologien nicht gewährleistet.

Somit ist eine durchgängige Betriebssicherheit der Maschinen nicht gegeben. Kombiniert mit den seit einigen Jahren obligatorischen Rückfahrkameras befindet sich auf den Maschinen nicht nur eine Vielzahl von Sensoren an den Außenseiten der Maschine; auch in der Kabine sind weitere Bildschirme notwendig. Diese schränken wiederum die Sicht des Bedieners auf sein Umfeld ein, nicht zu sprechen davon, ob die Person überhaupt in der Lage ist, bei den notwendigen kurzen Reaktionszeiten alle relevanten Informationen zu erfassen und zu verarbeiten - um letztlich adäquat und der Situation angemessen zu reagieren.

Diese Unsicherheit verhindert zudem eine weitere Automation und Autonomisierung der Arbeitsprozesse. Technisch wäre es bereits heute möglich, fahrende Baumaschinen, wie zum Beispiel Raupen, Gräder, Fertiger, Walzen, Fräsen und andere, vollautonom nach zuvor definierten Ablaufschemata bestimmte Arbeiten verrichten zu lassen. Diese können durchaus dynamisch aufgebaut sein. Aufgrund des vorhandenen Fachkräftemangels - der sich demographisch weiter ausweiten wird - ein Hemmnis, welches es durch sichere Assistenzsysteme zu durchbrechen gilt. Ebenfalls ein Problem ist die fehlende Rechtssicherheit beim Einsatz von Systemen zur Umgebungserkennung.

\subsubsection{Vorgehensweise und erste Ergebnisse}

Für das Projekt DigiRAB wurde das mobile Person Detection System (PDS) aus dem Bergbauprogramm der Firma Selectronic Funk- und Sicherheitstechnik GmbH ausgewählt. Es hat die Vorteile, dass nur ein Sensor pro Maschine benötigt wird und dieser mit geringem Aufwand nachrüstbar ist. Die Vermessung der eingeschränkten Sichtfelder ist vorteilhaft, bevor es zu einer Installation des Systems kommt. Dies ist besonders an Bestandsfahrzeugen zu empfehlen, zu denen es evtl. keine 3D-Daten mehr gibt oder auch weil sie während der Nutzungsphase oftmals den Arbeitsbedingungen entsprechend modifiziert wurden. Im Verlauf von DigiRAB wurden mehrere Baumaschinentypen (u. a., große und kleine Radlader, Bagger, Kompaktlader, LKWs) unter Baustellenbedingungen anhand neuester Normen durch Laser Scanning vermessen.

Das PDS basiert auf Radio Frequency Detection (RFID). Es erzeugt über die Antenneneinheit ein Feld mit einem codiertem 12 Bit Signal bei 7-9 kHz von maximal ca. $30 \mathrm{~m}$ Durchmesser. Somit hat es nach vorne und nach hinten ein Detektionsfeld von 
ca. $15 \mathrm{~m}$. Da sich das Feld aufgrund der Physik nicht als Kreis abbildet, entsteht an den Seiten ein Feld von ca. 11 m Entfernung (Verhältnis von ca. 1:1,35). Dieses Feld wird von der PDS-Software in drei Zonen unterteilt (frei konfigurierbar), und sobald der Personen-Sender in einer Zone detektiert wird, sendet er via UHF-Frequenz (ISM-Band) ein Datenprotokoll an die Fahrzeugeinheit zurück. Diese löst dann die entsprechende Reaktion am Fahrzeug aus.

Im Rahmen von DigiRAB wurde ein neues TFT Display von Grund auf entwickelt (Hardware/Software). Es wurde ein 4,3 Zoll Display verwendet, das auch unter Tageslicht gut lesbar ist. Bei der Entwicklung wurden alle für das Projekt erforderlichen Schnittstellen und ein interner Speicher berücksichtigt. Über diese Schnittstellen können die generierten Daten, die beim Eintreten einer Person in den Gefahrenbereich erzeugt werden, in Echtzeit weitergeleitet und/oder im internen Speicher abgelegt werden. Alle Daten können vollständig anonymisiert und frei von persönlichen Informationen gesammelt werden.

Die im Speicher abgelegten Daten, können einerseits über eine USB-Schnittstelle ausgelesen werden. Die Echtzeitdatenübertragung wird anhand einer Schnittstelle der Firma Topcon Deutschland Positioning GmbH realisiert, wo die Daten um die Geoposition per Global Navigation Satellite System (GNSS) ergänzt und zur Weiterleitung komprimiert werden. Ein solcher Datenstring besteht aus den folgenden Informationen: Personentag-ID (keine persönlichen Daten), Fahrzeug-ID, Zeitstempel, Detektionszone und Geoposition. Zur weiteren Verarbeitung werden die Datenstrings an einen Webservice von thinkproject übertragen.

Der Personen-Sender ist in einem robusten Gehäuse eingebaut. Die Akkuladung erfolgt über eine berührungslose QI-Technik (Induktion). Bei einer Detektion des Bausteins im Gefahrenbereich bekommt der Träger eine optische sowie eine akustische Warnung (optional ergänzt durch eine LED-Warnweste). Gleichzeitig wird auch der Maschinenführer optisch sowie akustisch über einen Buzzer oder Stimme gewarnt. Es wurden drei verschiedene Näherungszonen am Fahrzeug per Software-Konfiguration eingestellt, die je nach Bedarf auch unterschiedliche Reaktionen des Displays und der Baumaschine hervorrufen können. Denkbar ist hier ein Eingriff über das Bussystem der Baumaschine, um die Maschine proaktiv bei Gefahr zu verlangsamen oder gar zu stoppen. Dies wird bereits bei der momentanen Anwendung des Systems im UntertageBergbau praktiziert. Die Baumaschinenindustrie ist aufgefordert, dies auch bei Erd- und Straßenbaumaschinen zu ermöglichen.

Parallel zu dem oben beschriebenen System wurde ein zweites System auf 2,4 GHz Basis entwickelt und getestet. Der Hintergrund ist, dass jeder Funkwellenbereich sowohl Vor- als auch Nachteile besitzt. Das RFID System ist auf ca. $15 \mathrm{~m}$ für vorn und hinten beschränkt, wobei man im GHz-Bereich Reichweiten bis ca. $80 \mathrm{~m}$ realisieren kann. Dies bedeutet natürlich mehr Flexibilität für die Zonen. Ein Nachteil des zweiten Systems ist aber, dass es im Nahbereich (aufgrund von Reflexionen) unter $10 \mathrm{~m}$ keine genaue Detektion des Personen-Senders zulässt. Beide Funksysteme (kHz und $\mathrm{GHz}$ ) wurden erfolgreich getestet (Abb. 26.3 links). 

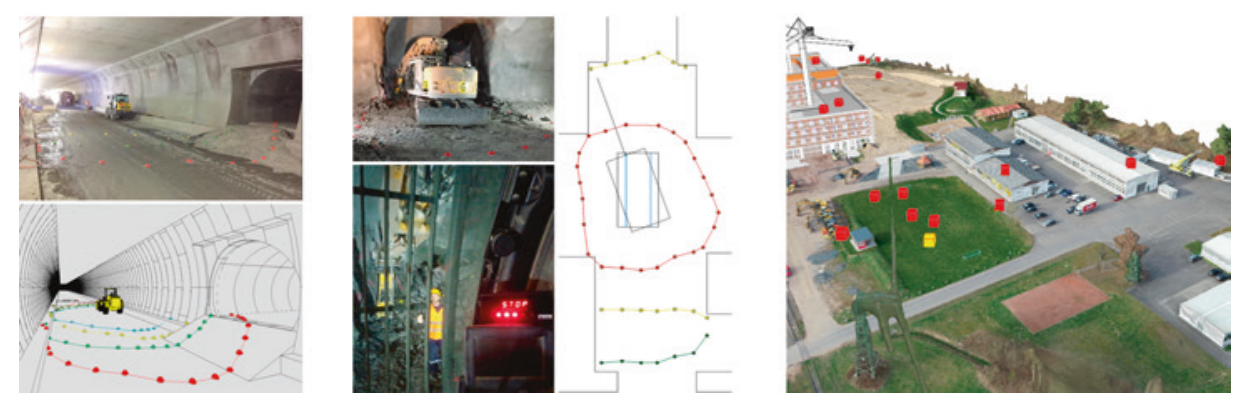

Abb. 26.3 Visualisierung der Warnzonen in einer Tunnelbaustelle (links und Mitte) [11-13] und anhand Hazard Cubes im georeferenzierten Modell der Demonstrator-Baustelle (rechts)

Um die von der entwickelten Technologie erzeugten Daten erfassen und auswerten zu können, hat thinkproject im Rahmen von DigiRAB einen Webservice entwickelt, in dem alle eingehenden Daten gesendet und gespeichert werden. Beim Auftreten eines Ereignisses (engl. close call event) werden die erzeugten Datenstrings an den Webservice übermittelt. Die Software DESITE MD verbindet sich über die Schnittstelle „Formulare“ mit dem Webservice. Der Benutzer hat in DESITE MD dann die Möglichkeit, einen Zeitraum auszuwählen, um die entsprechenden Gefahrenereignisse digital als Würfel (sogenannte „Hazard Cubes“) in DESITE MD zu visualisieren (Abb. 26.3 rechts). Jeder Würfel trägt die Informationen, welche über den Server von den Sensoren auf der Baustelle übermittelt wurden. Alle gefährdungsrelevanten Ereignisse der Bauphase können auf diese Weise dokumentiert und ausgewertet werden, um in Zukunft solche Gefahrensituationen vermeiden zu können.

Wenn der Benutzer sich in die Datenbank von DESITE eingeloggt ist (hierbei wird eine Kontrolle der Zugriffsberechtigung ausgeführt), greift der Algorithmus über die HTML-Datei von DESITE zuerst auf die Events von dem Webservice zu, die zu dem ausgewählten Zeitraum gehören. Die Daten werden im JSON-Format durch CURL oder REST API übertragen. Bevor die Events im DESITE-Projekt abgebildet werden, werden zwei wichtige Kontrollen ausgeführt. Zuerst wird überprüft, ob die eindeutige ID des Events im Projekt bereits vorhanden ist. Wurden die Events bereits in einer früheren Abfrage übermittelt, verhindert der Algorithmus, dass diese Events erneut erzeugt werden. Als Zweites wird überprüft, ob die GNSS-Koordinaten der übermittelten Events zu den Projektkoordinaten passen. Events, die außerhalb des Projektbereichs liegen, werden dementsprechend nicht übertragen. Diese Kontrolle ist insbesondere im Zusammenhang mit der Nutzung einer mobilen Anwendung (z. B. auf einem Tablet oder Smartphone) von Interesse, um zu verhindern, dass ein Benutzer mittels eines mobilen Endgerätes Daten zu einem falschen Projekt senden kann.

Wenn beide Kontrollen durchgeführt wurden, werden die Events in Form von Würfeln (Hazard Cubes) im digitalen Modell abgebildet. Die gespeicherten GNSS-Koordinaten 
werden mithilfe der DESITE APIs von GPS (WGS84) in lokale Modellkoordinaten umgewandelt. Bei den X, Y und Z-Koordinaten handelt es sich um den Ursprungspunkt des Würfels, der in DESITE MD in der 3D-Ansicht als "unit cube" erzeugt wird. Alle gefährdungsrelevanten Ereignisse der Bauphase können dokumentiert und ausgewertet werden, um in Zukunft solche Gefahrensituationen vermeiden zu können.

\subsection{Personalisiertes Lernen und Schulen (HB 3)}

\subsubsection{Ausgangslage}

Tausende Auszubildende von Baufirmen (in der Regel von kleinen und mittleren Unternehmen, KMU) beginnen jährlich eine mehrjährige Ausbildung in einem der angebotenen Bauberufe. Neben der Unterweisung in den Tätigkeiten im Umgang mit Baumaschinen (z. B. Anschlagen von Lasten an Krane), Werkzeugen (z. B. sachgemäßes Nutzen von Handgeräten), temporären Materialien (z. B. Auf- und Abbau von Gerüsten) und Baustoffen (z. B. Deklaration möglicher Gefahren), erfolgen auch Schulungen in den Bereichen des Arbeitsschutzes, insbesondere der Arbeitssicherheit und dem Gesundheitsschutz (z. B. Einhaltung von Gesetzen und Richtlinien).

\subsubsection{Motivation}

Zurzeit werden in den Ausbildungsstätten die Lehrmodule hauptsächlich in theoretischer Form anhand von (selbst) erstellten Schulungsunterlangen, gemäß den Ausbildungsrichtlinien geschult. Optional werden auch klassischer Frontalunterricht und Selbststudium kombiniert. Als Lernunterlagen stehen Bücher, digitale Dokumente und einfache 2D-Animationen zur Verfügung. Wegen des erheblichen finanziellen Aufwandes und des wenigen Lehrpersonals erfolgen in vielen Lehrmodulen nur selten Praxisschulungen. Die praktische Ausbildung erfolgt an speziellen Trainingsanlagen oder unter gesicherter Anleitung in produktiver Umgebung. In der produktiven Umgebung, besonders für den Bau komplexer Anlagen, können nur wenige Arbeitsschritte erprobt werden, da eine Fehlbedienung vermieden werden muss. Konsequenzen einer Fehlbedienung, können somit nur unzureichend praktisch vermittelt werden. Die wenigen Trainingsanlagen, die in zentralen oder internen Ausbildungsstätten verfügbar sind, ermöglichen nur die Erprobung von vorgegebenen Arbeitsschritten und sind mit erheblichen Einrichtungs- und Betriebskosten verbunden.

\subsubsection{Mixed Reality Anwendungen}

Klassische 3D-Anwendungen werden über Interaktion mit realen Elementen (z. B. Tastatur, Maus, Touchdisplay) gesteuert. In einer Virtual-Reality (VR)-Umgebung taucht 
der Nutzer vollständig in die Virtualität ein und steuert virtuelle Elemente über spezielle Controller. Augmented Virtuality (AV) bezeichnet die Art von Anwendungen, bei der reale Objekte in die Virtualität eingebracht werden. Dies kann in Form von Betriebsdaten sein oder durch Einbringung von weiteren haptischen Komponenten bis hin zur Aufnahme von realen Gegenständen über Kameras, die in die virtuelle Umgebung eingefügt werden. Die Bandbreite an Einbindungsmöglichkeiten umfasst dabei reale Interaktion mit virtueller Reaktion, virtuelle Interaktion mit realer Reaktion, sowie die virtuelle Abbildung realer Inhalte.

Mixed Reality (MR) Anwendungen [14] können genutzt werden, um die heutigen Probleme in Bezug auf den Mangel an realitätsnahen Trainingsumgebungen und die Simulation von kritischen Arbeitsaufgaben zu lösen. MR zielt in Anlehnung auf das konstruktivistische Lernparadigma darauf $a b$, eine möglichst realitätsnahe aktive Auseinandersetzung mit dem Lerngegenstand $\mathrm{zu}$ ermöglichen [15]. Wichtig für eine effektive und effiziente Anwendung von MR in der Aus- und Weiterbildung ist die Analyse bestehender Lernprozesse, das genaue Verständnis des Bedarfs einsatzfähiger Methoden inkl. technischer Lösungen, die zielgerichtete Entwicklung und die Erfassung des Mehrwerts. Im Folgenden werden diese erklärt und mögliche Anwendungsszenarien anhand von praktischen Beispielen vorgestellt.

\subsubsection{Vorgehensweise}

Im Rahmen von DigiRAB wurden von der Ruhr-Universität Bochum eigene VR- und AVSimulationen entwickelt. Diese vermittelten den Anwendern arbeitssicherheitsspezifische Aspekte auf Baustellen, insbesondere das erfolgreiche Erlernen des sicheren Umgangs mit Baumaschinen und handgeführten Geräten (u. a. Winkelschleifern). Für die Evaluation der VR-Simulationen wurde ein Evaluationskonzept entwickelt und geprüft. Das Konzept bezieht dabei sowohl die formative Evaluation während des Entwicklungsprozesses von Simulationen mit ein als auch abschließende Evaluationen mit dem potenziellen Endnutzerkreis. Besondere Aufmerksamkeit der formativen Evaluation galt den technischen sowie inhaltlichen Aspekten der Simulation. Zum einen wurden iterative Tests in Zusammenarbeit mit der Hilti AG sowohl mit Experten des Systems als auch mit unerfahrenen Nutzern durchgeführt, damit möglichst breit gefächerte Rückmeldungen erzeugt werden konnten. Die Ergebnisse als auch die gemachten Beobachtungen im Umgang mit der Technik wurden als Änderungshinweise dokumentiert und im Nachgang umgesetzt. Dabei ging es im Wesentlichen darum, dass der Realitätsgehalt der VR-basierten Lernumgebung stimmig ist und keine irritierenden Abweichungen von der üblichen Erfahrungswelt auf Baustellen beinhaltet. Als herausfordernd hat sich hierbei vor allem herausgestellt, die notwendigen technischen Änderungen mit der erzählten Story innerhalb der Simulation konsistent zu halten, da kleine Änderungen im Ablauf einzelner Erzählbausteine direkte Konsequenzen auf die logischen Abläufe innerhalb der Simulation haben konnten. Erst wenn nach ein paar Iterationen ein Stand der Simulation erzeugt wurde, der sowohl inhaltlich auch technisch 
den gewünschten Kriterien entspricht, konnte ein Testlauf der abschließenden Evaluation zum Lernerfolg mit realen Probanden durchgeführt werden.

Im Mittelpunkt der abschließenden Evaluation stand vor allem die Prüfung des Einsatzes von VR-Simulationen als Ergänzung zu vorhandenem Schulungsmaterial und Schulungssystemen. Um zentrale Gütekriterien in der Evaluation zu erfüllen, wurden insbesondere Standardinstrumente der Gebrauchstauglichkeitsprüfung (Usability) sowie des Nutzerempfindens (User Experience) verwendet [16]. Eingesetzte Instrumente der Evaluation sollten sein: Fragebogen zur Erfahrung mit Arbeitssicherheit auf Baustellen, zur Arbeit auf Baustellen und zu den in der Simulation dargestellten Lerninhalten abfragt; semi-strukturierten Interviews sowie Beobachtung.

\subsubsection{Ergebnisse}

In den entwickelten virtuellen Lernumgebungen werden authentische Lernsituationen mit Hilfe von Ansätzen des situierten Lernens unter Verwendung von VR und AVSimulationen [17] dargestellt (Abb. 26.4). Anhand realistischer Anwendungen im Hochbau werden mögliche Gefahren und Risiken wirklichkeitsnah veranschaulicht (u. a.: Kranlasten, Baustellenverkehr, zu enge Arbeitsbereiche). Um diesen Effekt zu verstärken, werden die Simulationen über echte handgeführte Geräte gesteuert.

Das Evaluationskonzept wurde in einer exemplarischen Studie zum sicheren Umgang mit Baumaschinen und Winkelschleifern erprobt. Die Ergebnisse der durchgeführten Studie zeigen eine durch die Teilnehmer gute bis sehr gut bewertete Usability sowie einen positiven Lerneffekt bei den Teilnehmern [20]. Der beobachtete Lerneffekt ohne weiteres Fremdeinwirken kann hier potenziell als eine Grundlage zur Weiterbildung für migriertes Fachpersonal dienen, da digitale Lehrinhalte lediglich einmal übersetzt werden müssten und im Gegensatz zu „learning off the job“-Weiterbildungsangeboten
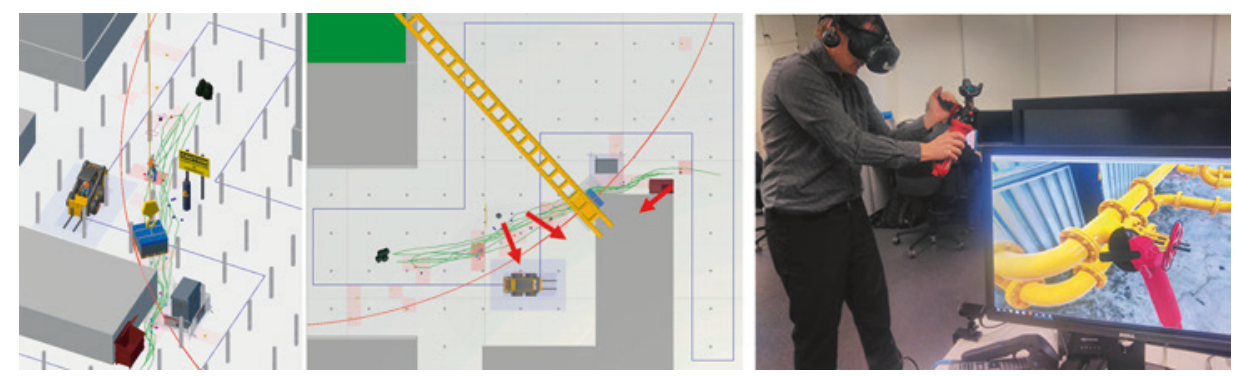

Abb. 26.4 Echtzeit-Datenanalyse von Beinahe-Unfällen zwischen Bauarbeitern und Baumaschinen und anderen vorab definierten Gefahrenstellen während einer Schulung in VR (links) [18] und Einsatz von handgeführten Maschinen in VR anhand realer Bauszenarien (rechts) [19] 
mit Lehrkräften nicht an Personal gebunden sind. Innerhalb der Studien hat sich zudem gezeigt, dass die Teilnehmer während der Interviews wiederholt ihre Entscheidungen in der Simulation reflektiert und gewisse Aspekte auch auf reale Erlebnisse aus ihrem bisherigen Berufsleben bezogen haben. Die Möglichkeit, das erlebte Szenario in den Simulationen noch einmal zu besprechen, hat somit reflektive Lernprozesse ausgelöst. Reflektives Lernen zeichnet sich dadurch aus, dass neu erlebte Geschehnisse gedanklich mit vergangenen Erfahrungen verglichen und bewertet werden und sich aus diesem Prozess wiederum neue Entscheidungen, Verhaltensweisen oder Wissen ergeben können. Dem Einsatz der Simulationen ohne Lehrpersonal kann ein entsprechend durch Lehrkräfte unterstützter Einsatz gegenübergestellt werden. Dabei können die Anwender der VR-Simulationen das erlebte Szenario gemeinsam mit den Lehrkräften besprechen und es werden lernförderliche Reflexionsprozesse angestoßen [21].

Eine Auswahl der wichtigsten Ergebnisse zur exemplarischen Erprobung der VRSimulation mit Baumaschinen und Winkelschleifern:

- Die Simulationen boten sichere Lernumgebungen an, wobei 2 von 14 Teilnehmern an Motion Sickness (Schwindelgefühl) litten.

- Es wird deutlich, dass sowohl die entwickelte Simulation als auch die eingesetzte Hardware zur Steuerung innerhalb der Simulation leicht bedienbar waren und das System insgesamt mit einer guten bis sehr guten Usability bewertet wurde. Selbst ohne Vorerfahrungen konnte das System problemlos verwendet und die Aufgaben in der Simulation gelöst werden.

- Es sind mit dem Simulationsspiel verbundene Lerneffekte zur Arbeitssicherheit anhand der statistischen Analyse der Ergebnisse des Fragebogens erkennbar. Die Interviews zeigen, dass sich die meisten Teilnehmer nach der Simulation als sicherer im Umgang mit Winkelschleifern einschätzen und die dabei relevanten Hintergründe zur Arbeitssicherheit verstehen.

- Die User Experience wurde wie auch die Realitätsnähe der Darstellungen in der Simulation von den meisten Teilnehmern äußerst positiv bewertet. Außerdem äußerten mehrere Teilnehmer den Wunsch nach weiteren Szenarien.

Eine wesentliche weitere Erkenntnis ist, dass nachhaltiger Lernerfolg anhand von Verhaltensänderungen in der Realität gemessen werden muss. Daraus lassen sich in Zukunft interessante Lernkonzepte des kontinuierlichen Lernens und Fortbildens mittels der Virtuellen Realitäten entwickeln. So sollen Anwenderinnen und Anwender die besonders riskanten Stellen und Situationen auf Baustellen kennenlernen, um damit Unfällen vorzubeugen. Und zwar auf genau der Baustelle, auf der sie später arbeiten oder für die sie als Arbeitsschutzplaner verantwortlich sind. Nach dem Baukastenprinzip dienen BIM Modelle als Grundlage für die Repräsentation der Baustelle in der virtuellen Realität. Damit die computergenerierte Baustelle einigermaßen naturgetreu aussieht, müssen sie Oberflächentexturen, Schatten und Umgebungsinformationen wie Häuser, Straßen und Bäume 
beinhalten. Auch die fahrenden Baumaschinen mit typischen oder realen Bewegungsabläufen dürfen nicht fehlen. Durch Geräusche wird das virtuelle Szenario noch realistischer. Die Bauarbeiter werden virtuell (in einer sicheren Umgebung, die Fehler zulässt) geschult, jeweils nach den individuellen Bedürfnissen oder Arbeiten, die auf der Baustelle zu erledigen sind. Planer können damit Gefahrenquellen vorab lokalisieren und eliminieren (s. HB1). Nach dem TOP-Prinzip können weitere erforderliche Maßnahmen erfolgen und getestet werden, z. B. die Installation zusätzlicher Absperrungen, um getrennte Laufwege von Personal am Boden nahe an Baumaschinen vorzugeben.

\subsection{Zusammenfassung}

Das Forschungs- und Entwicklungsprojekt DigiRAB entwickelte smarte Prozesse und Technologien für das sichere Arbeiten auf Baustellen. Im Verlauf des Projekts hat es sich im Wesentlichen auf mehrere Anwendungsszenarien in der Digitalisierung des Arbeitsschutzes fokussiert: (1) Digitales Planen zur Prävention von Gefahrenstellen, (2) Proaktives Warnen, Melden und Auswerten und (3) Personalisiertes Lernen und Schulen. Wesentliche Innovationen, die erfolgreich erprobt wurden sind:

- Automatisiertes regelbasiertes Prüfen von Sicherheitsregeln anhand von Building Information Modeling (BIM) zur präventiven Planung von Baustellensicherheitseinrichtungen

- Proaktives Erkennen, Warnen, Melden, und Visualisieren von Beinahe-Unfällen (Mensch-Maschine-Interaktion) in Echtzeit

- Personalisiertes Schulen und Lernen in der Gefahrenerkennung durch Erweiterte und Virtuelle Realitäten

\section{Projektpartner und Aufgaben}

- Ruhr-Universität Bochum - Lehrstuhl für Informatik im Bauwesen, Lehrstuhl für Informations- und Technikmanagement

Individuelle Schulungskonzepte auf Basis digitaler Modelle und smarter Technologien

- Ed. Züblin AG - Direktion Mitte

Digitale Planung des Arbeitsschutzes auf Baustellen

- Topcon Deutschland Positioning GmbH

Smarte Arbeitsmittel für das sichere Arbeiten auf Baustellen

- ceapoint aec technologies $\mathbf{G m b H}$

Präventive Warnungen in der vernetzten Baustelle

- Selectronic Funk- und Sicherheitstechnik GmbH

Proaktive Warnsysteme und Analyse von sicherheitsrelevanten Informationen 


\section{Literatur}

1. Teizer J, Melzner J (2019) "Neue Wege zur Integration von BIM, IoT und Lean Construction in der Bauingenieurausbildung", Bauingenieur, Springer, S 19-25

2. Zhang S, Teizer J, Lee J-K, Eastman C, Venugopal M (2013) Building information modeling (bim) and safety: automatic safety checking of construction models and schedules. Autom Constr, Elsevier 29:183-195

3. Teizer J, Melzner J, Wolf M, Golovina O, König M (2017) Automatisierte 4D-Bauablaufvisualisierung und Ist-Datenerfassung zur Planung und Steuerung von Bauprozessen. Springer, Bauingenieur, S 129-135

4. Schwabe K, Teizer J, König M (2019) Applying rule-based model-checking to construction site layout planning tasks. Autom Constr, Elsevier 97:205-219

5. Lingard H (2013) Occupational health and safety in the construction industry. Constr Manage Econ 31(6):505-514

6. Hinze JW, Teizer J (2011) Visibility-related fatalities related to construction equipment. J Saf Sci, Elsevier 49(5):709-718

7. Golovina O, Perschewski M, Teizer J, König M (2019) Algorithm for quantitative analysis of close call events and personalized feedback in construction safety. Autom Constr, Elsevier 99:206-222

8. Teizer J (2015) Safety 360: surround-view sensing to comply with changes to the ISO 5006 earth-moving machinery test method and performance criteria, 32nd ISARC, Oulu, Finland

9. Golovina O, Perschewski M, Teizer J, König M (2018) Predictive analytics for close calls in construction safety, 35th ISARC, Berlin, Germany

10. Netzwerk Baumaschinen (2019) Personen-/Objekterkennung, Warnung in Gefahrenbereichen. http://www.netzwerk-baumaschinen.de/material/download/nwBMA_Personen-Objekterkennung.pdf. Zugegriffen: 01. Dez 2019

11. Teizer J (2015) Magnetic field worker proximity detection and alert technology for safe heavy construction equipment operation, 32nd ISARC, Oulu, Finland

12. Golovina O, Teizer J, Rauth F, König M (2018) Proaktive Magnetfeldtechnologie zur Unfallvermeidung an Baumaschinen. Springer, Bauingenieur, S 52-67

13. Rauth F, Golovina O, Teizer J, König M (2019) "Erhöhung der Arbeitssicherheit im Tunnelbau durch proaktive Kollisionsvermeidung", Taschenbuch für den Tunnelbau 2019. Wiley, Berlin, S 175-220

14. Teizer J, Wolf M, König M (2018) Mixed Reality Anwendungen und ihr Einsatz in der Ausund Weiterbildung kapitalintensiver Industrien. Springer, Bauingenieur, S 73-82

15. Piaget J (1954) The construction of reality in the child. Basic Books, New York, US

16. Brooke J (1996) SUS: a „quick and dirty“ usability scale, Usability evaluation in industry. Taylor and Francis, London

17. Wolf M, Teizer J, Ruse JH (2019) Case study on mobile virtual reality construction training, 36th ISARC. Banff, Canada

18. Golovina O, Kazanci C, Teizer J, König M (2019) Using serious games in virtual reality for automated close call and contact collision analysis in construction safety, 36th ISARC. Banff, Canada

19. Wolf M, Bükrü S, Golovina O, Teizer J (2020) Using field of view and eye tracking for feedback generation in a virtual power facility safety training. Construction Research Congress, Tempe, Arizona, USA

20. Bükrü S, Wolf M, Böhm B, König M, Teizer J (2020) „Augmented Virtuality in Construction Safety Education and Training.“ European Group for Intelligent Computing in Engineering Conference (EG-ICE), Berlin, Germany, July 1-4, 2020, S. 115-124 
21. Solberg A., Hognestad JK, Golovina O, Teizer J (2020) „Active Personalized Training of Construction Safety Using Run Time Data Collection in Virtual Reality.“ 20th Intl. Conference on Construction Application of Virtual Reality (CONVR), Middlesbrough, UK, S 19-30

22. Li B, Schultz C, Melzner J, Golovina O, Teizer J (2020) „Safe and Lean Location-based Construction Scheduling“. 37th International Symposium on Automation and Robotics in Construction, Kitakyushu, Japan, October 27-28, 2020. https://doi.org/10.22260/ ISARC2020/0195.

23. Schultz C, Li B, Teizer J (2020) „Towards a Unifying Domain Model of Construction Safety: SafeConDM.“ European Group for Intelligent Computing in Engineering Conference (EGICE), Berlin, Germany, July 1-4, 2020, S. 363-372

24. Neve HH, Wandahl S, Lindhard S, Teizer J, Lerche J (2020) „Determining the relationship between direct work and construction labor productivity in North America: Four decades of insights." J Constr Eng Manag 146(9):04020110. https://doi.org/10.1061/(ASCE)CO.1943-7862.0001887

Open Access Dieses Kapitel wird unter der Creative Commons Namensnennung 4.0 International Lizenz (http://creativecommons.org/licenses/by/4.0/deed.de) veröffentlicht, welche die Nutzung, Vervielfältigung, Bearbeitung, Verbreitung und Wiedergabe in jeglichem Medium und Format erlaubt, sofern Sie den/die ursprünglichen Autor(en) und die Quelle ordnungsgemäß nennen, einen Link zur Creative Commons Lizenz beifügen und angeben, ob Änderungen vorgenommen wurden.

Die in diesem Kapitel enthaltenen Bilder und sonstiges Drittmaterial unterliegen ebenfalls der genannten Creative Commons Lizenz, sofern sich aus der Abbildungslegende nichts anderes ergibt. Sofern das betreffende Material nicht unter der genannten Creative Commons Lizenz steht und die betreffende Handlung nicht nach gesetzlichen Vorschriften erlaubt ist, ist für die oben aufgeführten Weiterverwendungen des Materials die Einwilligung des jeweiligen Rechteinhabers einzuholen.

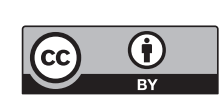

